\title{
BMJ Open Quality Using safety culture results to guide the merger of four general practices in the UK
}

\author{
Alistair Martin Lockwood (D , ${ }^{1}$ Joshua Proulx, ${ }^{2}$ Matthew Hill, ${ }^{3}$ Joanna Pendray ${ }^{4}$
}

To cite: Lockwood AM, Proulx J, Hill $\mathrm{M}$, et al. Using safety culture results to guide the merger of four general practices in the UK. BMJ Open Quality 2020;9:e000860. doi:10.1136/ bmjoq-2019-000860

- Additional material is published online only. To view please visit the journal online (http://dx.doi.org/10.1136/ bmjoq-2019-000860).

Received 21 October 2019 Revised 6 February 2020 Accepted 1 March 2020
Check for updates

(c) Author(s) (or their employer(s)) 2020. Re-use permitted under CC BY-NC. No commercial re-use. See rights and permissions. Published by BMJ.

${ }^{1}$ St Austell Healthcare, St Austell, UK

${ }^{2}$ Safe and Reliable Healthcare, Evergreen, Colorado, USA

${ }^{3}$ Anaesthetics, Plymouth Hospitals NHS Trust, Plymouth, UK

${ }^{4}$ Southwest Academic Health Science Network (SWAHSN), Exeter, UK

Correspondence to Dr Alistair Martin Lockwood; alistair.lockwood@nhs.net

\section{ABSTRACT}

Background The rapid merger in a crisis of three GP practices to incorporate the patients from a neighbouring closing surgery, led to the redesign of primary care provision. A deliberate focus on patient safety and staff engagement was maintained throughout this challenging transition to working at scale in an innovative, integrated and collaborative GP model.

Method 3 cycles of a staff culture tool (Safety, Communication, Organizational Reliability, Physician \& Employee burn-out and Engagement) were performed at intervals of 9-12 months with structured feedback and engagement with staff after each round. The impact of different styles of feedback, the effect of specific interventions, and overall changes in safety climate and culture domains were observed in detail throughout this time period.

Results Strong themes demonstrated were that: there was a general improvement in all culture domains; specific focus on teams that expressed they were struggling created the most effective outcomes; an initial lack of trust of the management structure improved; adapting and tailoring the styles of feedback was most efficacious; and burn-out scores dropped progressively. A unique observation of the rate at which different modalities of safety climate and culture change with time is demonstrated.

Conclusion With limited time, resources and energy, especially at times of crisis or change, the rapid and accurate identification of which domains of 'culture' and which teams required the most input at each stage of the journey is invaluable. Using this tool and prioritising patient safety, enables rapid and effective positive change to the culture and shape of expanding practices. It affirms that new models of working at scale in GP can be positively embraced with improvements in safety culture, if this is deliberately focused on and included in the transition process.

\section{INTRODUCTION}

In 2014, Polkyth Surgery unexpectedly collapsed leaving 9000 patients without a General Practitioner (GP). This was due to limited local GP workforce, difficulty in GP partner recruitment and subsequent financial difficulties. In response to this St Austell Healthcare (SAH) was formed in 2015 after the merger of three neighbouring practices in St Austell to also incorporate the patients from Polkyth Surgery. St Austell is a town of approximately 40000 people in central Cornwall, with high levels of deprivation and a high disease burden. ${ }^{1}$ The merger was significantly challenging due to the low numbers of GPs available locally, the different models of working and infrastructure across the original practices, and the significant crisis in which this occurred. However it enabled the opportunity to redesign primary care provision across the town with a change from the four legacy practices working independently and serving 7000-9000 patients each, to a single more collaborative, integrated and innovative practice providing services to 32000 patients across four sites.

In December 2015 SAH became one of 15 rapid test sites for the National Association of Primary Care's (NAPC's) Primary Care Home Pilot. ${ }^{2}$ Its principles have guided the development of a new model of care in $\mathrm{SAH}$, prioritising collaborative working and closer cross-sector alliances to improve health and well-being for the community. SAH is now a NAPC Primary Care 'show case' site, has been included as an example of future models of care in a recent national review of general practice $^{3}$ and is featured in a King's Fund ${ }^{4}$ report on the spread of the primary care home model. The principles adopted closely match all the elements of the recent national GP contract, ${ }^{5}$ which is encouraging GP practices to form networks of 30-50 000 patients and broaden the range of clinicians they employ.

The practice has evolved to now run 'total triage' for all routine and urgent care work, currently operates across four sites, and is building strong links with community and secondary care teams in the locality. The staffing model is broad, embracing multiple multidisciplinary team (MDT) members including a dedicated visiting service run by paramedics, an acute Hub for all same day work, social prescribers, pharmacists, an integration nurse, Musculoskeletal (MSK) and 
mental health clinicians. We have approximately 100 staff, 11 GP partners with list sizes of approximately 3500 patients per eight-session GP.

The merging of the practices, with the incorporation of Polkyth Surgery, was a time of considerable uncertainty for staff and the local population, and the GPs were looking for ways to support this process as it occurred. In the early months of the merger, one of the executive GP partners was participating in Patient Safety Officer training with the South West Academic Health Science Network (SWAHSN) and realised the potential benefit of focussing on safety climate and culture, particularly in times of such instability and stress, and noted that more studies have been encouraged. ${ }^{67}$ The Safety, Communication, Organizational Reliability, Physician \& Employee burn-out and Engagement (SCORE) staff survey tool was offered as part of the course and had the potential to support the practice through the merger and in particular would increase the engagement with all members of the staff to enable their contribution, be responsive to their suggestions to the practice design, and facilitate their involvement in the improvements and integration of practice staff.

There is very little published experience of use of safety climate staff surveys in General Practice in the UK, with most of the focus being in secondary care. Studies have looked at assessing safety climate across various GP sites as cross-sectional surveys at a single point in time ${ }^{7-9} \mathrm{~A}$ previous Randomised Control Trial (RCT) ${ }^{10}$ across multiple stable GP practices involving specific safety interventions over a 9-month period demonstrated no improvement in safety culture domains. There are a range of tools that could be used ${ }^{11} 12$ and discussions about creating practical ways of using them and improving work in GP on safety climate. ${ }^{713}$

The aim of this study was a deliberate focus on patient safety and staff engagement throughout this transition to working at scale in a new GP model. The impact of different styles of feedback and engagement with staff, the effect of different specific interventions, and the rate at which different elements and modalities of safety climate and culture change with time were observed in detail.

\section{METHOD}

The SCORE tool is an internationally recognised and validated $^{14}$ staff survey tool, which was provided and hosted by an external provider (www.safeandreliablecare.com). It was supported financially by SWAHSN/Patient Safety Collaborative. It is currently being used to survey all National Health Service (NHS) maternity and neonatal departments in England as part of the Maternal and Neonatal Health Safety Collaborative, ${ }^{15}$ a major NHS Improvement project.

SCORE is a 45-question tool with the answers on a 5-point Likert Scale. The domains are broken down into improvement readiness (six questions), team working (seven questions), safety climate (seven questions), local leadership (seven questions), burn-out climate (five questions), personal burn-out (five questions), and work/life balance (eight questions).

Prior to starting the survey we communicated with all staff telling them about why we were doing the survey, that it would be anonymous and that the teams would be debriefed about the results to celebrate the good work and opportunities for improvement.

\section{Administration of score survey}

The survey was run as an electronic survey that was distributed to all staff by email and takes approximately 10 min to complete. It typically runs for 6 weeks or until a response rate of greater than $60 \%$ is obtained.

The survey is anonymous and the only demographic that it records is job title. In order to preserve the anonymity of staff only data with four or more responses for a role type are released. Role types with less than four responses are merged into other role types or a separate 'other' category.

The survey has been taken at intervals of 9-12 months for three full cycles starting in February 2016. This covered the entire process of the practices merging then rapidly evolving to new models of working across multiple sites, including the development of a broad MDT and integration with community teams.

The results are analysed by Safe and Reliable Healthcare and the results are available as an interactive webbased tool or as a pdf document. Some sample questions are included in the online supplementary appendix.

\section{Patient and public involvement}

We did not involve patients or the public in our work, as the survey tool is designed for use by staff teams and not patients.

\section{Feedback and debriefing of results}

The practice teams had a debriefing session where the results were discussed to allow further understanding of the reasons driving the results and a collaborative approach with individual teams to influence the overall direction of the practice. This feedback and debriefing were undertaken in a range of styles, all involving regular face-to-face meetings and ongoing conversations with one lead GP and relevant team managers.

The minimum response rate for reporting results from SCORE is $40 \%$, and at least $60 \%$ is the recommended minimum for any given work setting to provide a representative consensus view from respondents.

\section{RESULTS}

\section{Response rates}

The response rates for each cycle of administration of the SCORE survey were greater than $60 \%$ (table 1). This allows an accurate image of the workplace climate to be created. 
Table 1 Response rates for the three surveys

\begin{tabular}{lll}
\hline Survey period & Number of responses & Response rate \\
\hline Feb 2016 & 60 & $64 \%$ \\
Dec 2016 & 70 & $64 \%$ \\
Dec 2017 & 83 & $76 \%$ \\
\hline
\end{tabular}

\section{Domain scores}

The domain scores (figure 1) show an improvement in all domains over the period of the study. An increase of $10 \%$ is considered to be a significant increase and all apart from the teamwork domain exceed or approached this. Note, in this figure higher scores in both burn-out domains indicate lower levels of burn-out.

Different staff groups showed different levels of improvement (figure 2). Results are presented here as group level differences over time, however, we also analysed the results at the respondent level over time and found the same patterns of findings as with the group level.

\section{Improvements in local leadership and overall shared vision} domains

A progressive improvement in local leadership domains was demonstrated (figure 3) contrasting a slower improvement in overall shared vision domains (figure 4)

\section{Burn-out domains}

A general trend to show reduction in burn-out (figure 5) and improved work-life balance were noted.

\section{'Would I feel safe to be treated here'}

A key question within the survey is whether individual staff members would 'feel safe being treated here as a patient'. This demonstrated an improvement (figure 6) with a significant reduction of negative responses and an overall positive trend.

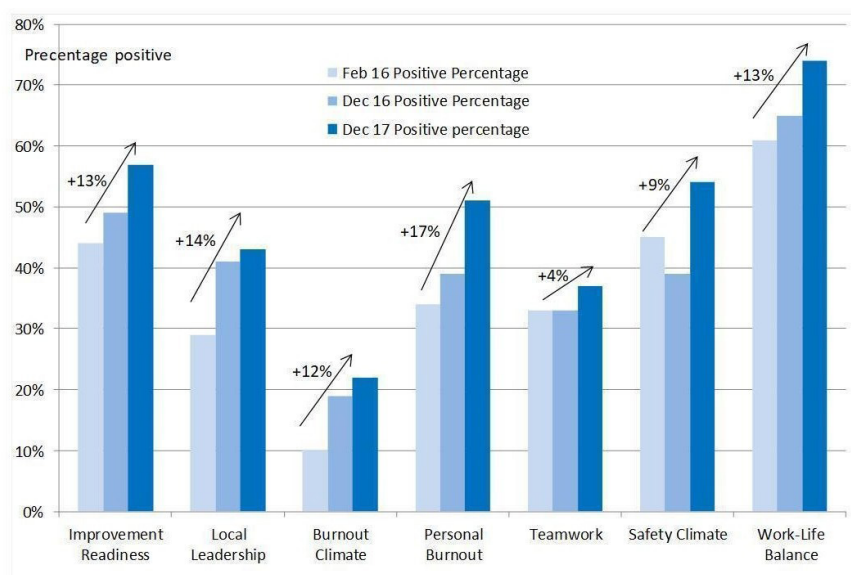

Figure 1 Improvements in safety culture domains for all groups.

\section{DISCUSSION}

\section{Summary}

The past 3 years have seen big changes for the 32000 patients and over 100 staff of SAH. Against a background of challenging times in the NHS, the transition to a new way of working has not always been smooth. General practice is being encouraged to work at a larger scale and to collaborate as part of the GP networks that sit as the foundation stones of the new GP contract. ${ }^{5}$ This process of transition is challenging and potentially daunting, particularly when it is not through choice, is complex and uncertain, and is on top of already substantial clinical workloads. Despite these challenges, we have been able to demonstrate improvements in the measured safety culture within SAH.

The key messages from our observations include:

\section{Psychological safety of staff}

The minimum response rate for reporting results from SCORE is $40 \%$, and at least $60 \%$ is the recommended minimum for any given work setting to provide a representative consensus view from respondents. There were response rates of $>60 \%$ for the three surveys, and this improved with time. There are a number of possible reasons for this that include: the importance given to the survey by the local leadership; active encouragement to complete the survey; a clear explanation of the tool's rationale and use; as a voluntary survey the emphasis given to the psychological safety of the staff including guaranteed anonymity; and that staff would be involved in discussions about the results to identify areas of good work and opportunities to improve. The improved rates of response with consecutive rounds reflects a progressively increasing level of trust and confidence in the process by staff.

We noticed that the process of continual structured and informal dialogue with individuals, teams and managers is time-consuming but essential to gain momentum in cultural change. This was best done led by one GP who consistently encouraged the broader partners and teams to engage and participate.

\section{Improved domain scores}

A general progressive improvement in all domains was achieved over the course of the surveys. This is likely in part to be due to the substantial practice-wide efforts to make the merger successful from all staff groups involved but will also reflect the deliberate focus on staff culture and very targeted responses to feedback that were acted on from one survey to the next. This is very encouraging for anyone who is in a situation of current crisis or change. Yet it must be taken in the context of this practice using the process of feedback, debriefing and proactive response to problems, in the setting of a team pushing hard to innovate and develop.

It is also likely that this level of improvement was achievable as it is a fully merged practice with autonomy to change and develop. It has no external factors directly 

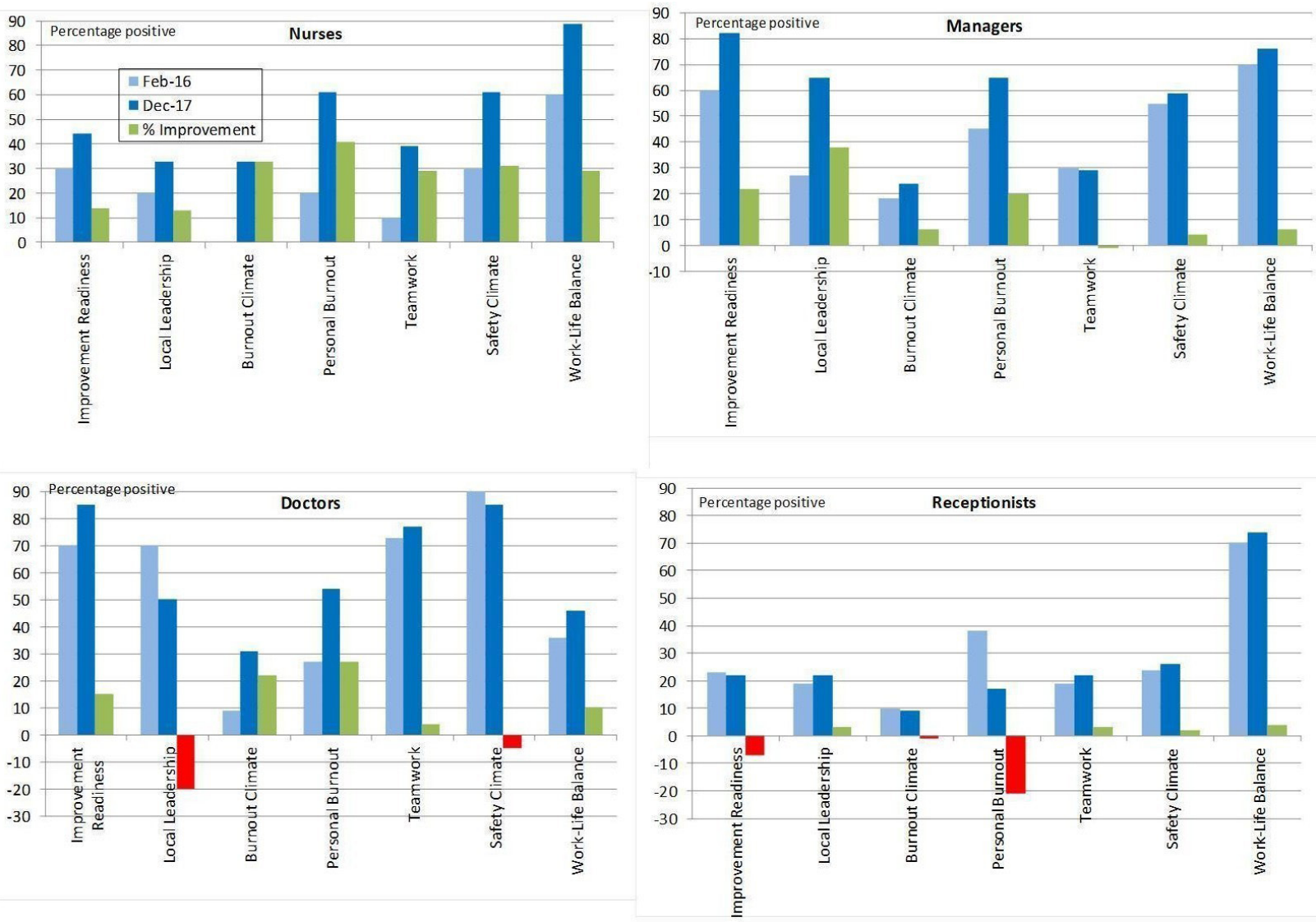

Figure 2 Improvements in safety culture domains for individual groups.

interacting with its internal operational matters and has a very clear vision and cohesive partnership model. If this were a department in a larger trust or organisation, change would likely have been slower as there is so much direct impact from neighbouring departments and the larger trust. Likewise, in a federated GP model (where practices do not fully merge) this pace of change and the effectiveness of culture tools is likely to be slower and

\section{Local Leadership Historical Comparison}

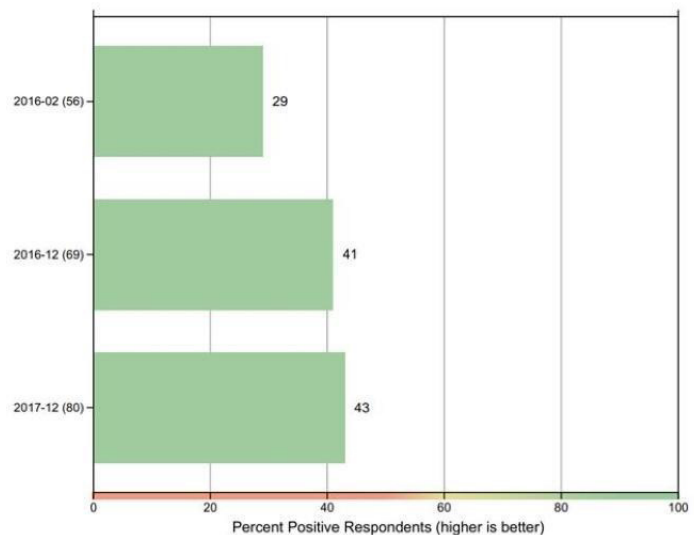

Source Data: Dec 2017

Institution: St Austell Healthcare

Work Setting(s): All Work Settings

Position(s): All Positions

Figure 3 Local leadership scores for all groups. less complete as the operational alignment and shared teamwork will be less combined and integrated. Similarly, change is likely to be slower or less complete in a GP setting with possibly more pressure, or less energy and focused shared vision to improve.

\section{Improvement in leadership domains}

There has been an improvement in the perception of local leadership since the initial survey which showed a

The values of the organisations leadership are the same values that people in this work setting think are important.

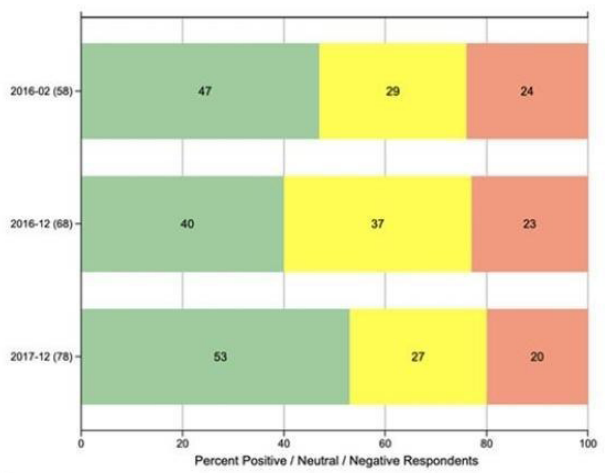

Source Data: Dec 2017 Institution: St Austell Healthcare
Work Setting(s): All Work Settings Work Setting(s): All Work Settings
Position(s): All Positions

Figure 4 Shared values with the executive vision for all groups. 
Personal Burnout Historical Comparison

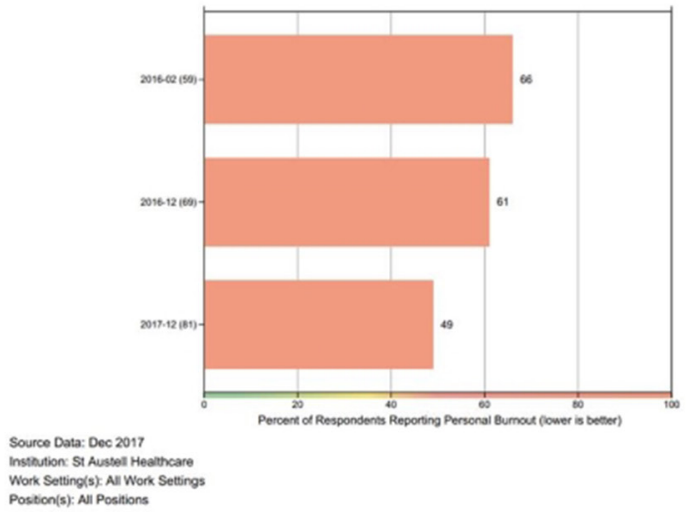

Work-Life Balance Historical Comparison

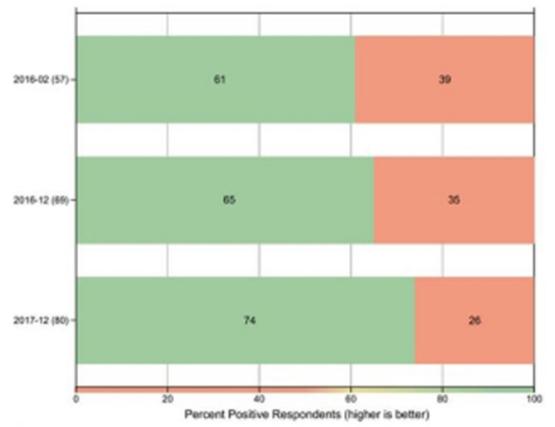

Source Data: Dec 2017 Institubion: SI Austell Heathicare Work Setting(s) All Work Sostitions): NI Postions

Figure 5 Improvement in personal burn-out domain and work-life balance.

relatively low score of $29 \%$. Initial feedback from staff indicated a lack of confidence and engagement with both their local line management and the executive running of the practice. The decisions to merge were made by the partners not the staff initially and hence it is understandable that early results indicated a lack of understanding of the overall vision and a lack of confidence in hastily established management structures.

The traditional GP partnership model makes a more formalised management structure more difficult, with staff often not used to the more structured approach usually found in larger businesses or organisations. Historically staff would approach any partner, often undermining another member of staff or another partner if need be, with a lack of clear shape as to who an individual should approach with a problem or feedback. Supporting and encouraging a reliable shape and line management structure was a positive move for staff and helped clarify the role of a partner versus the management shape.

I would feel safe being treated here as a patient.

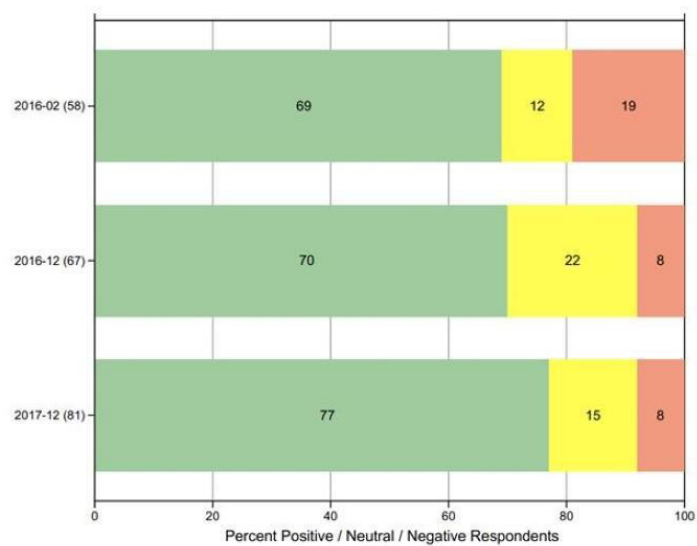

Source Data: Dec 2017

Institution: St Austell Healthcare

Work Setting(s): All Work Settings

Position(s): All Positions

Figure 6 Overall responses to 'I would feel safe being treated here as a patient'.
The debriefing of this data enabled teams to discuss how they wanted to be led, encouraged discussion about the organisational values, and therefore helped drive and shape the existing management structure and improved scores. This matches hospital studies ${ }^{16}$ that have demonstrated that safety climate and organisational culture are positively linked, with strategies including promotion of group orientation and reducing hierarchy being helpful. Our staff clearly indicated they wanted a definite idea of who they could approach and that any feedback was acted on. Re-enforcement and refinement of this shape has been required on a continual basis, but all members of the team have responded positively to this. Its success, however, is dependent on feedback being regularly acted on and responded to in a transparent and timely manner.

It has been evident that there has been an overall slow improvement in the shared vision of the staff in the overall direction of the practice. The staff leading the change (mostly the GPs and managers) led the scores in all domains from the start as they were the 'innovators' making the decisions, while it has taken time for the rest of the staff to fully engage with this overall vision. Previous studies have shown managers to score higher than other staff, ${ }^{8}$ and as we demonstrate this may include the GP partners as well as managers. ${ }^{9}$ Awareness of the difference in perceptions of managers/GP partners and other staff is key if a strong safety culture is to develop. ${ }^{9}$

The practice is still multisite working and it is likely that until the aspired one-site solution is built there will always be a limit to how much a 'silo-mentality' of working and improvement of shared vision can be achieved. This may also partly explain the slow improvement in teamwork domains compared with other areas of more rapid improvement. It has been very difficult to continually, clearly reflect and explain a changing overall direction in such a rapidly evolving local health economy and diversifying practice to all staff. We have found it imperative to attempt to regularly and consistently communicate the broader vision and direction. Improvement in scores will probably accelerate with a one-site solution and further 
integration with community teams, yet this is going to take time to bring to fruition.

\section{Variation in team improvements}

In the first round the nursing team indicated a significant issue with the local leadership and engagement of the whole team on multiple domains. They reported that it was not clear what the role of two newly appointed nurse matrons was and, as they had come from different legacy practices they did not understand each other's working practices. This was expressed as suspicion of the staff who they did not know. In order to understand the responses, it was decided to offer an interview with each nurse, which was conducted by the lead GP. We were cognisant of the importance of psychological safety for the employees and took efforts to create an environment where they could safely voice their thoughts.

All ideas were collated and then passed back to the nurses, including the new matrons, in a group meeting, and a shared plan of action was decided on by the whole nursing team. Among themselves they prioritised the most important issues. This led to the establishment of a six weekly regular nurse training morning, which was so successful it was spread to all teams to meet in protected time to train and grow as teams. It also led to the re-enforcement and encouragement of the matron line management structure and showed significant improvement in all domains that persisted into round 3.

Without the insights from the survey it would not have been obvious that the nursing group needed targeted support at the early stages of the merger, as so much else was going on. It allowed the prioritisation and deployment of our finite resource of time and energy to be focused on this team, with significant success. The intervention was guided by the round 1 results and focussing on one team interestingly led to changes across many other teams as well as a result of focussed work with them. It helped break down a 'silo mentality' we suspected but could not clarify and enabled the roots of effective multisite and MDT working to begin. This has now spread to the way we approach our integration with the community. The general lack of engagement, shared vision and contentment is highly likely to have continued across all teams if we had not had the tools to enable identification of the teams and domains requiring most input.

In contrast, the reception team who we have struggled to engage with due to the complexity and diversity of their role, has not shown progress and continues to feed back a lack of engagement and support, with less improvement in all domains. They are currently the prime focus of improvement and engagement work.

\section{Styles of feedback and debriefing}

Different approaches were successful in different settings in terms of debriefing. Adapting the approach to different staff groups ensured the active participation of staff in the process and their involvement in identifying what and how to improve.
For example, in the first round the nursing team lacked a clear leadership shape and confidence in the matron roles, and hence individual work with the nurses was very effective as described above. In later rounds we have found it more successful to get teams to collate feedback and meet as a group, with the line manager in the room. This is in response to scores for confidence in local leadership rising consistently and, in a desire, to reinforce the line management shape. This has been really useful in recent work with the reception team as it is evident that the cultural issues affecting their team are not at a reception and local management level but a higher managerial and partnership level with how information flows from 'local' line managers to the partners/exec and back. This would not have been evident if we had continued to focus on an individual level, and anything that undermined the management structure would likely have made the overall confidence in the organisation worse.

We have recognised that detailed reflection at the time of debriefing on what the confidence of the staff is in the local and executive leadership, and where in the journey of organisational change the practice is, will enable effective and targeted methods of communicating and improving practice.

\section{Burn-out}

Personal burn-out scores improved across the practice as a whole and this was mirrored by improvements in worklife balance. Also those individuals with more defined workloads had better stress and work-life balance scores, whereas GPs with unlimited administrative loads, receptionists with constant patient contact and secretaries with continually replenished dictation tasks, did not score so well.

Very interesting patterns emerged about a difference in personal burn-out and the perception of burn-out of others (burn-out climate). Personal burn-out scores were consistently lower across all groups compared with the perception of burn-out in others. It is evident we portray more stress externally than we perceive in ourselves.

A targeted intervention between rounds 2 and 3 enabled all staff to engage in two half-day stress management and resilience-training sessions provided by a clinical psychologist from the local NHS Improving Access to Psychological Therapies (IAPT) providers, Outlook South West. This was offered in direct response to these scores. The first session focused on general principles of personal stress management. The second session was tailored by the attendees of the first session, and at their preference focussed on a range of subjects including, understanding negative and positive mindsets and relationships at work, and dealing with difficult conversations. There was a demonstrable accelerated improvement in burn-out scores that correlate with this intervention. This helps confirm the positive benefits to a practice of providing time and resources on high-quality psychology support to all its own staff, including practical tools on personal stress management and techniques for managing stressful 
situations particularly around challenging patients or colleagues. We now employ two Community Psychiatric Nurses (CPNs) as part of our practice who are keen to further develop the inhouse psychology support to staff.

This burn-out data show that effective use of larger practice sizes or 'networks' (aspiring to 30-50 000 patients) involving incorporation of a broad MDT team, can improve work-life balance and reduce burn-out. This contrasts other studies that show attempts at 'leaner' working in healthcare can increase burn-out especially for physicians, ${ }^{17}$ and that larger GP practices, particularly in areas of higher deprivation had lower overall culture scores, reflecting possible burn-out. ${ }^{8}$

Of importance, the work pattern for GPs shifts quite significantly to higher list sizes and more administrative burden, but with a strong effective team around them to support them in this. Despite the work pressure continuing to rise in the NHS, the data suggest burn-out and work-life balance improve, and that new ways of doing General Practice are efficient and sustainable.

\section{Implications for research and/or practice}

This is an observational study done throughout a stressful merger process. Multiple confounding factors within the rapidly evolving practice limit being able provide definitive causality of the survey tool itself. However, the observation of the cultural change over time, the efficacy of interventions attempted and the overall impact of the deliberate focus on staff engagement using a validated survey tool can be demonstrated.

Very little is published on the deliberate use of regular staff surveys on the development of culture within expanding health organisations, especially general practice. With significant potential benefits of using these tools, further avenues of research focussing on specific elements of cultural change within the transition to, and working within new models of working at scale in GP and other healthcare settings, would be valuable.

Our sample involved only employees of the practicefuture work should include the broader community 'network' MDT team and patients.

\section{CONCLUSION}

This study is the first to demonstrate the effective use of a validated staff safety climate survey, repeated regularly over a period of time to support the merger of four General Practices. With limited time, resource and energy, especially at times of crisis or change, the rapid and accurate identification of which domains of 'culture' and which teams required the most input at each stage of the journey is invaluable. This tool, and the processes of debriefing and acting on data, have proved incredibly useful in producing the most rapid and effective positive change to culture and the shape of the developing practice.

The regular process of debriefing specific data in an honest and transparent way, enabling staff to 'own' their own data and then shape the actions that come from it, has shifted the whole culture and conversation that goes on. This has helped create an effective, dynamic practice and management structure, which is constantly being refined.

Our learning and experience from this survey tool, and importantly how to use this data in developing a team and culture, is likely be supportive to other similar situations, and a range of other settings. It provides a road map to shape cultural change within expanding organisations. It is a unique observation of the rate at which different elements and modalities of safety climate and culture, change in this time of rapid practice evolution. It also affirms that new models of working at scale in GP can be positively embraced with improvements in safety culture, if this is deliberately focused on and included in the transition process.

In times of crisis or change, if there is a strong, shared vision of improving patient care, with a deliberate focus on engagement of all members of a team, this can unite to drive significantly positive overall results. And it makes it an enjoyable, sustainable place to work.

Contributors AML: principal author, led the survey process within the practice, and coordinated writing and completion of the article. JPr: principal statistical analysis and data production, contribution to writing the paper. $\mathrm{MH}$ and JP: Organisation and interpretation of the initial survey process, contribution to writing the paper.

Funding The surveys were funded by the Southwest Academic Health Science Network (SWAHSN).

Competing interests None declared.

Patient consent for publication Not required.

Provenance and peer review Not commissioned; externally peer reviewed.

Data availability statement Data are available upon reasonable request.

Open access This is an open access article distributed in accordance with the Creative Commons Attribution Non Commercial (CC BY-NC 4.0) license, which permits others to distribute, remix, adapt, build upon this work non-commercially, and license their derivative works on different terms, provided the original work is properly cited, appropriate credit is given, any changes made indicated, and the use is non-commercial. See: http://creativecommons.org/licenses/by-nc/4.0/.

ORCID iD

Alistair Martin Lockwood http://orcid.org/0000-0003-3813-1084

\section{REFERENCES}

1 Cornwall Council. St Austell health and social demographics, 2017. Available: https://www.cornwall.gov.uk/media/27613266/staustellcna-profile.pdf [Accessed Aug 2019].

2 NAPC. Overview of primary care home projects, 2019. Available: http://napc.co.uk/primary-care-home [Accessed Aug 2019].

3 UK Government. Gp partnership review case studies: business models. Available: https://assets.publishing.service.gov.uk/ government/uploads/system/uploads/attachment_data/file/770913/ gp-partnership-review-final-report-case-studies.pdf [Accessed Aug 2019].

4 King's fund review of NAPC Primary Care Home Spread, 2019. Available: https://napc.co.uk/wp-content/uploads/2019/06/Insightsfrom-the-Spread-of-the-Primary-Care-Home.pdf [Accessed Aug 2019].

5 NHS England. Investment and evolution: a five-year framework for GP contract to implement the NHS long term plan, 2019.

6 Proudfoot J, Jayasinghe UW, Holton C, et al. Team climate for innovation: what difference does it make in general practice? Int $J$ Qual Health Care 2007;19:164-9. 
7 Kirk S, Parker D, Claridge T, et al. Patient safety culture in primary care: developing a theoretical framework for practical use. Qual Saf Health Care 2007;16:313-20.

8 Bell BG, Reeves D, Marsden K, et al. Safety climate in English general practices: workload pressures may compromise safety. $J$ Eval Clin Pract 2016;22:71-6.

9 de Wet C, Johnson P, Mash R, et al. Measuring perceptions of safety climate in primary care: a cross-sectional study. J Eval Clin Pract 2012;18:135-42.

10 Hoffmann B, Müller V, Rochon J, et al. Effects of a team-based assessment and intervention on patient safety culture in general practice: an open randomised controlled trial. BMJ Qual Saf 2014;23:35-46.

11 Curran C, Lydon S, Kelly M, et al. A systematic review of primary care safety climate survey instruments: their origins, psychometric properties, quality, and usage. J Patient Saf 2018;14:e9-18.
12 Hoffmann B, Domanska OM, Albay Z, et al. The Frankfurt patient safety climate questionnaire for general practices (FraSiK): analysis of psychometric properties. BMJ Qual Saf 2011;20:797-805.

13 Mannion R, Davies H. Understanding organisational culture for healthcare quality improvement. BMJ 2018;363:k4907.

14 Sexton JB, Adair KC, Leonard MW, et al. Providing feedback following leadership WalkRounds is associated with better patient safety culture, higher employee engagement and lower burnout. BMJ Qual Saf 2018;27:261-70.

15 NHS Improvement, 2019. Available: https://improvement.nhs.uk/ resources/maternal-and-neonatal-safety-collaborative [Accessed Aug 2019].

16 Singer SJ, Falwell A, Gaba DM, et al. Identifying organizational cultures that promote patient safety. Health Care Manage Rev 2009;34:300-11.

17 Hung DY, Harrison MI, Truong Q, et al. Experiences of primary care physicians and staff following lean workflow redesign. BMC Health Serv Res 2018;18:274. 